\title{
BMJ Open Quality Implementing a triage tool to improve appropriateness of care for children coming to the emergency department in a small hospital in India
}

\author{
Neha Joshi, ${ }^{1}$ Rakhi Wadhwani, ${ }^{2}$ Jitender Nagpal, ${ }^{1}$ Saru Bhartia ${ }^{2}$
}

To cite: Joshi N, Wadhwani R, Nagpal J, et al. Implementing a triage tool to improve appropriateness of care for children coming to the emergency department in a small hospital in India. BMJ Open Quality 2020;9:e000935. doi:10.1136/ bmjoq-2020-000935

- Additional material is published online only. To view, please visit the journal online (http://dx.doi.org/10.1136/ bmjoq-2020-000935).

Received 29 January 2020 Revised 24 June 2020 Accepted 25 August 2020

\section{Check for updates}

(c) Author(s) (or their employer(s)) 2020. Re-use permitted under CC BY-NC. No commercial re-use. See rights and permissions. Published by BMJ.

${ }^{1}$ Paediatric, Sitaram Bhartia Institute of Science and Research, New Delhi, Delhi, India

${ }^{2}$ Quality, Sitaram Bhartia Institute of Science and Research, New Delhi, Delhi, India

Correspondence to

Saru Bhartia;

saru.bhartia@sitarambhartia.org

\section{ABSTRACT}

Background In 2015, senior consultants at Sitaram Bhartia Institute of Science and Research saw several sick children in their outpatient clinics for which they had been seen in the emergency department the previous day. These children seemed to require admission but were sent home. This prompted us to review the paediatric care provided in our emergency department.

Methods A multidisciplinary team was formed to run this improvement initiative. Review of literature suggested that establishing a triage system around a prevalidated triage tool would help us deliver more appropriate care. The South African Triage Scale was selected and adapted. Interventions With the aim of delivering appropriate care to at least $50 \%$ of children, a series of sequential interventions were tested using the improvement methodology of Plan-Do-Study-Act (PDSA) cycles, an approach recommended by the Institute for Healthcare Improvement. Learnings from the PDSA cycle of the previous intervention helped decide the subsequent change idea. The interventions included training in use of tool, increasing nurse staffing levels, using team huddles as feedback opportunities, introducing nurse reminders, reducing non-productive work, developing local leadership and training a restricted group of locum paediatricians. Qualitative and quantitative information was analysed to retain or reject change ideas.

Results At baseline only $16 \%-17 \%$ of children were receiving appropriate care. The sequential changes resulted in a gradual improvement to a median of $63 \%$ of children receiving appropriate care by the end of 20 months.

Conclusions We succeeded in establishing a paediatric emergency triage system and culture in the given setting through a unique enriching experience. We worked on removing systemic barriers and facilitating change while facing several unexpected outcomes. A sustained iterative approach may be the best way to achieving significant improvement in difficult settings like ours.

\section{INTRODUCTION}

\section{Problem description}

In March 2015, senior paediatricians at Sitaram Bhartia Institute of Science and Research (SBISR) noted that they were seeing many sick children in their outpatient clinics who had received medical treatment in the emergency department (ED) the previous day. Clinical assessment of these children revealed that many of these patients were sent home even though they required hospital admission. These observations prompted us to evaluate paediatric care in the ED.

We analysed paediatric emergency consults over 4 weeks. On arrival of a child in the ED, the nurses telephonically communicate with the paediatrician working in the hospital requesting a visit. We realised in this communication the nurse did not inform the paediatrician about the level of urgency (routine, emergency or urgent) when reporting the child's arrival. This hindered the doctors' ability to prioritise the new patient compared with their other work in the wards. It was also worrisome to observe that for some of the consults, paediatric evaluation by different doctors led to different clinical advice for similar conditions. For example, infants with similar severity of respiratory distress were kept under observation by some doctors while sent home by others on inhaler medication.

\section{Available knowledge}

Acute care of ill children is a global public health issue, and there is tremendous scope to improve quality of care in hospitals at all levels especially in low and middle-income countries. ${ }^{1}$ Possible areas of improvement include initial triage, emergency care, assessment, inpatient treatment and monitoring. ${ }^{2}$ Many deaths in hospitals can be prevented if sick children are identified soon after their arrival in the health facility and treatment is started immediately. This can be facilitated by a triage tool for all children coming to the hospital to identify those needing immediate emergency care. ${ }^{3}$ Triage instruments establish a system of clinical management that ensures patients are seen in order of their clinical need rather than in order of arrival. It also helps to manage patient flow safely when clinical needs exceed capacity. Emergency Nurses Association and American College of 
Emergency Physicians also believe that quality of patient care would benefit from implementing a standardised ED triage scale and acuity categorisation process.

Many scoring systems exist for paediatric emergency triage purposes. Most existing triage scores were developed in western countries and include multiple physical and laboratory variables making them cost and labour intensive and difficult to implement in the ED. Some of the existing triage systems for paediatric emergencies are Manchester Triage Scale, ${ }^{4}$ Emergency Severity Index, ${ }^{5}$ Canadian Triage and Acuity Scale, ${ }^{6}$ Australasian Triage Scale, ${ }^{7}$ WHO Emergency Triage and Treatment Scale, ${ }^{8}$ and so on. Processing a high volume of patients quickly and performing a sensitive triage that identifies the sickest children in a low to middle-income country setting is more complex due to scarcity of resources. ${ }^{9}$

\section{Rationale}

It was hypothesised from the review of literature that implementation of a triage tool would help us deliver appropriate care. The tool could help our team assign clinical priority, standard assessments and take medically appropriate decisions on admission, observation or discharge. But with paediatric emergency medicine still in its infancy in India and standardised practices still under evolution we had to find a triage tool that could work in our setting. Most of the paediatric emergencies in India, including in tertiary care hospitals, are managed by paediatric residents who are also responsible for managing non-emergency conditions. ${ }^{10}$ Dedicated triage teams and medical personnel especially trained in emergency medicine are not the norm in most centres. ${ }^{11}$ Given these limitations we reviewed existing tools to identify a triage tool suitable for use by paediatric doctors and emergency nurses.

We reviewed the literature and identified the South African Triage Scale (SATS) to be appropriate for our setting. SATS is a physiology and symptom-based scale that categorises complaints and symptoms into coloured themes. It was designed by a multidisciplinary team of doctors, nurses and paramedics and validated in public, private and prehospital settings. ${ }^{12}$ It has been reported to be useful in other South Asian countries which have healthcare set-ups similar to India. ${ }^{13-15}$

\section{Specific aim}

To use a triage tool to provide appropriate care to at least $50 \%$ of paediatric patients coming to the ED in a period of 12 months. We classified a child as receiving appropriate care if the admission status (admission, observation or discharge) assigned by the junior paediatricians, or locum at the end of the emergency stay was correct by the clinical health record (CHR) and triage tool audit.

\section{METHODS}

\section{Context}

SBISR, a 70-bedded, non-profit hospital in Delhi provides paediatric care to 1500 children in the outpatient clinic and an additional 100 in the ED every month.
The paediatric department comprises two senior and six junior consultants. All these doctors run outpatient clinics, inpatient and emergency care. Round-the-clock care to admitted and emergency patients is ensured by a roster.

The ED at SBISR is staffed with a resident medical officer (RMO) and nurses at all times. The RMO screens the adult patients coming to the ED and initiates treatment. He treats these patients in consultation with senior consultants from various medical disciplines. However, for paediatric patients the nurse telephones the paediatrician to come and treat the child. The paediatrician then visits the ED, examines the child and documents the child's complaints, examination details, treatment given and medical advice to the child in an emergency CHR. This record has a separate section for the nurses in which they record vitals and the initial assessment done by them. At the end of the consultation a copy of this record is handed over to the patient while the original is retained by the hospital. The RMOs and paediatricians work in two shifts-morning (09:00-17:00) and night (17:00-09:00), while the nurses work in three shifts-morning $(08: 00-$ 14:00), evening (14:00-20:00) and night (20:00-08:00).

\section{Interventions}

Our intervention was the implementation of a paediatric triage tool. We formed an improvement team which consisted of two senior paediatricians, a quality consultant, one emergency nurse and a quality officer. The senior paediatricians modified some of the symptom presentations mentioned in SATS to incorporate our local presenting complaints to enable context-specific assessments ${ }^{15}$ and created a paediatric triage tool.

This tool is a colour-coded document in which a child's complaints are assigned to one of three categories-emergency (E-red), urgent (U-yellow) or routine (R-green). The nurse receiving the child categorises the child $(\mathrm{E} / \mathrm{U} / \mathrm{R})$ and informs the junior paediatrician telephonically about the category of the child. The nurse also follows category-specific standard management instructions (eg, If a child comes with gasping breaths, is cold, pale and lethargic, only responding to pain, the nurse immediately needs to start the oxygen and then calls the paediatrician. She then needs to prepare the resuscitation and intravenous cannulation tray). The paediatrician needs to promptly reach the ED, examine the patient and use the tool to calculate a final Triage Early Warning Score (TEWS) that provides guidance on whether to admit, observe or discharge the child (online supplemental appendix 1).

Once the tool was adapted the senior paediatrician gave individual training sessions to each junior paediatrician, RMO and emergency nurse. A training video was also added to the emergency computers for the staff to refer in case of need. The tool was then integrated to the existing process flow of emergency care (online supplemental appendix 2). To measure appropriate care we focused only on the paediatrician entries in the tool as 
they were directly linked with care advice (admission/ observation/discharge). To interpret appropriate care the completeness and correctness entry was considered necessary.

As a precursor to the implementation, the team decided to test the feasibility of the tool.

\section{Intervention 1: application of triage tool in different shifts,}

November and December 2015

Plan-Do-Study-Act (PDSA) 1: We first applied the triage tool on receiving a patient to the ED to determine whether the tool was easy to use and to evaluate the time taken to use it. This PDSA was run in November 2015 during the morning shift on a single patient. We interviewed the paediatrician to understand the level of difficulty and time taken while using the tool. The interview revealed that the paediatrician found the tool easy to use and that it took an average of $3 \mathrm{~min}$ to fill the tool. We then gradually expanded the use of tool to all paediatric emergency consults in the morning shift. We then decided to test the tool in the evening and night shifts with another PDSA.

PDSA 2: We tested if we could spread the use of the tool to all paediatric emergency consultations in all shifts (morning, evening and night) by running a PDSA through December 2015. It was important to check the feasibility in the evening and night shifts because there were significant differences in staffing patterns (two nurses in the morning shift compared with one in the evening and night shifts) and workload during the three shifts. We evaluated the expanded tool usage by interviewing the nurses and doctors and by directly observing care. We learnt that the tool was valued for its role and was easy to use. However, interviews also revealed that there was a need of additional nursing staff in the evening and night shifts to ensure that the tool was filled because when the doctors had limited nursing help they were busy with additional clinical work and were reluctant to fill the tool. The team therefore got approval for additional nursing staff in the ED for these shifts.

While qualitative and subjective evaluations indicated that the tool was easy to use and appreciated by all, quantitative data for November and December 2015 revealed that the paediatricians completely filled the tool for only $32 \%$ and $41 \%$ of their emergency consults and filled the tool correctly (as per audit of clinical records) for just $18 \%$ and $17 \%$ leading to only $16 \%$ and $17 \%$ of patients receiving appropriate care in these 2 months.

\section{Interim review}

To review the perplexing situation the team met to identify possible barriers and brainstorm ideas to support the use of the tool. The following themes emerged in the review:

- Paediatricians suggested that regular feedback regarding their tool application would be helpful.

- They also shared that they were forgetting to fill the tool and would need regular reminders in the initial stages of implementation.
- They also mentioned that the tool was an added documentation and would be hard to fill during busy times. The team decided to address the concerns raised by the paediatricians and came up with multiple change ideas to resolve them. The following PDSA cycles were thus initiated sequentially to test these change ideas.

\section{Intervention 2: testing daily huddles to give feedback to} paediatricians on errors in triage tool application, January 2016 PDSA 3: The paediatric department has daily huddles during shift change to discuss admitted patients. Data from monthly audits on incorrect entries were shared with individual paediatricians during the huddles. For example, if the paediatrician used the table for $<3$ years old when assessing a 5-year-old child, the feedback would be given during the huddle. We saw significant improvement with this change. The paediatricians completed the tool in $76 \%$ of cases, correctly filled them $55 \%$ of the time and provided appropriate care to $48 \%$ of the patients. With significant improvement in care we decided to continue with feedback on the triage tool in February.

PDSA 4: To our surprise appropriate care fell to $33 \%$ as paediatricians completed the tool for $62 \%$ and correctly used the tool for only $36 \%$ of their emergency consults the following month. Conversations with some of them revealed that feedbacks were increasing being perceived as criticism of their performance and therefore disliked. We therefore rejected this change and explored other opportunities to aid paediatricians in transforming their practice to use the tool correctly.

\section{Intervention 3: using nurse-led reminders to paediatricians for} filling the triage tool completely, March 2016

PDSA 5: The workflow for paediatric emergency consults was revised such that the nurses after filling their section of the tool hand it to the paediatrician with a polite request about thorough tool application. During this PDSA we conducted daily meetings with nurses to seek feedback on their reminder efforts. We also had discussions with paediatricians to get their opinion on this intervention. Analysis revealed that while senior nurses reminded the paediatricians the junior nurses hesitated due to prevailing hierarchical dynamics. Even though it was originally their idea, the paediatricians despised being reminded and hence this intervention was rejected. Meanwhile, the percent of children receiving appropriate care remained unchanged (36\%).

Intervention 4: simplification of CHR to create time for tool application, April 2016

PDSA 6: To address the issue of additional documentation we decided to review the documentation work done by staff. We found that the pre-existing CHR had extensive documentation and the additional triage tool application further added to time spent towards record keeping. It was decided to simplify the CHR which the paediatricians had to fill along with the triage tool. The CHR was reformatted from an open-ended question format which 
required a lot of writing to one which had check boxes to tick. This also reduced duplicate documentation (information that was already being noted in the nurse's notes was deleted from the doctor's documentation). We took feedback from all paediatricians as well as direct observations during consults to collate qualitative data regarding utility of this change. Qualitatively, the team highly appreciated and valued the time saved for clinical interactions over paperwork.

However, appropriate care remained at $36 \%$, completely filled tool at $69 \%$ and correctly filled at $39 \%$. Though this change did not improve appropriate care we continued with the simplified record as it saved time which could be judiciously used for clinical interactions.

\section{Interim review}

At this point, we decided to reassess our gains and challenges. We reached out to paediatricians individually. Some paediatricians expressed the difficulty of a heavy load in the ward which amounted to rushed application of tool. Conflicting prioritisation between triage documentation and clinical attention to the child and their anxious family was another cited reason. We spent April and May exploring an idea of engaging the RMOs on using the triage tool at times when the paediatrician was busy. We decided to test this intervention through a PDSA in June.

\section{Intervention 5: feasibility and effectiveness of engaging RMOs} for tool application during a busy paediatrician work shift for uninterrupted tool use, June 2016

PDSA 7: A senior paediatrician trained the RMOs on how to use the tool. It was agreed that if there were more than three paediatric case arrivals, back to back at a given time or if the paediatrician was busy with a sick child in the hospital and unable to undertake emergency consult, the RMO would replace the paediatrician's role in using the tool during the emergency consult. To maintain patient safety, it was also decided that the RMO could consult the paediatrician telephonically like they did with other specialty experts. Daily morning meetings were organised (emergency staff, quality officer and paediatrician) to monitor adherence.

When we discussed with the RMO, nurses and paediatricians we identified a number of challenges with this change. The RMOs felt less capable and confident than the paediatricians in assessing children correctly and nurses shared that parents wanted the paediatricians to see their children. Paediatricians perceived that RMO's telephonic dependency on a paediatric consult did not relieve any burden during busy times. The change idea was hence rejected. In this period, the completeness, correctness and appropriate care all decreased to $54 \%$, $31 \%$ and 27\%, respectively, in June 2016.

\section{Interim review}

July and August witnessed a further drop in appropriate care to $20 \%$ and $19 \%$, respectively. We investigated the drop and found that the number of paediatric patients coming to the ED increased more than twofold during these months due to seasonal infections. This had resulted in new challenges. The nursing team observed delays in supply of stationery and late recruitment of additional manpower. Any administrative changes like change in staff duties, ordering medicines, consumables or even ordering stationery in the ED required approval of the nursing and paediatric department causing unnecessary delays in day-to-day management. This led us to explore local leadership in the ED. A volunteer from the ED, a senior nurse, also an early adopter of our tool, was identified as our triaging supervisor. This leader was a single point of contact for all stakeholders participating in triaging-related processes and was authorised to undertake administrative decisions at local level pertaining to same. We tested the idea of local leadership with PDSA 8.

Intervention 6: promoting an early adopter, a nurse, to a triaging supervisor, September 2016

PDSA 8: In this PDSA we tested whether increased autonomy given in local decision-making would help in improving logistics and proper tool use. During September, the senior nurse ensured adequate supplies like stationery, forms, photocopy machine, and so on were available. She promptly addressed day-to-day logistical issues. She anticipated and modified the emergency nursing staff roster to account for busy shifts. She reallocated non-clinical responsibilities undertaken by nursing staff to other support staff such as housekeeping, enabling prioritisation to clinical care and assistance to doctors in demanding shifts. She facilitated additions to drug and consumable inventories and modified reorder levels based on paediatric prescriptions. All these activities did not require approval by paediatric head or the nursing superintendent. With this change we hoped that direct supervision and timely closure of triaging toolrelated tasks would help team members perform their roles.

Discussion with stakeholders revealed that having a supervisor was highly valued as it improved work efficiency and helped in promptly addressing hurdles. Due to this change the tool was completely filled for $70 \%$ and correctly filled for $46 \%$ of the patients leading to $43 \%$ appropriate care. This was significant improvement from the preintervention period and hence the change was retained.

The triage supervisor shared with the team that the pool of locum paediatricians (new paediatricians hired for an interim period when there is shortage of hospital staff) were consistently applying the tool incorrectly and suggested that if we could identify only a selected number of locum paediatricians to do additional duties so that it would become easy to train them for the triage tool. The team thought it was a good idea and we tested it for the coming 2 months. 
Intervention 7: retaining the triaging supervisor to coordinate all triage-related requirements and create a pool of select locum paediatricians, October 2016

PDSA 9: This intervention was tested with a PDSA in October 2016. With this we wanted to test whether having a senior nurse as a local leader along with training a dedicated pool of locum paediatricians would help in further improving the appropriateness of care. In October, the paediatric team identified a pool of three locum paediatricians who would do additional duties when required in the coming month. During October, these three locums were trained before their shifts for the triage tool.

With increased engagement and regular training, the locums complied with the correct filling of the tool leading to increase in appropriate care. These data corroborated that having a dedicated pool of locums did not adversely impact reliable care. For October and November appropriate care was $44 \%$ and $42 \%$, respectively.

The team decided to continue with having the senior nurse as a triage supervisor, having a dedicated pool of locum paediatricians and a simplified medical record for the coming months. All PDSA cycles are summarised in table 1 for clarity.

\section{Measures}

We first measured the completeness of triage tool (patient category, scores for each vital parameter, total TEWS). In cases where the triage tool was complete we measured correctness-if the triage tool and CHR were consistent with each other (ie, Was the patient categorised correctly, score calculated correctly and status consistent with the score?). Where the tool was completely and correctly filled, the percentage of patients who received 'appropriate care' was measured. We classified a child as receiving 'appropriate care' if the admission status (admission, observation or discharge) was deemed appropriate by review of CHR and triage tool documents. This was our primary outcome measure. CHR and triage tool review was conducted in at least $50 \%$ of all emergency cases every month.

\section{Analysis}

We used run charts to analyse our quantitative data. We used the first 10 months to calculate the baseline median. We used the run chart rules proposed by Anhøj and Olesen $^{16}$ and defined a shift as a run of eight or more points on one side of the median. When we identified a shift, we used the eight data points that constituted the shift to calculate the new median.

We also analysed qualitative information collected through feedback from individual stakeholders, direct observations and group discussions. Information through these meetings, discussions and observations helped the team take a decision to either reject or retain an intervention. These data also guided the team for future interventions which were tested through incremental PDSA cycles which have been summarised in the Interventions section.

\section{RESULTS}

A run chart for appropriate care is presented in online supplemental appendix 3. We tested various interventions (annotated in graph) through several PDSA cycles during the initial phases and aimed at appropriate care for not less than $50 \%$ of patients. Following the implementation of our last change idea in September 2016 we have achieved our goal by providing appropriate care to median of $63 \%$ of patients (online supplemental appendix 3). We also are completely and correctly filling the tool for $86 \%$ and $64 \%$ of patients, respectively, since the implementation of our last change idea (online supplemental appendix 4).

\section{DISCUSSION}

\section{Summary}

Triage instruments facilitate stratification of sick paediatric patients to the ED and guide a timely advice on admission, observation or discharge. In the absence of pre-existing triage system we identified the need of a reliable and standardised decision-making tool. We therefore applied a prevalidated triage tool to deliver appropriate care. We selected a tool with established reliability for use by multidisciplinary staff and adapted it to address our regional needs. To interpret appropriateness of care we relied on measuring complete and correct (using clinical audit) triage tool application.

Aiming to provide appropriate care to our patients we trained our staff and implemented the tool as our main intervention only to realise that its application was not merely training dependent. The experience of the journey of sequential incremental interventions while incorporating subjective feedback and monitoring quantitative outcomes will be useful for anyone attempting work in a similar setting. While the study is unique the lessons learnt and inferences drawn can be applied across settings.

\section{Interpretation}

Interventions were tested and then implemented in a sequential manner to improve proper tool application to provide appropriate care. An intervention was retained if it was successful in removing logistic barriers or addressing emotions based on qualitative data. Impact of the intervention was parallelly analysed quantitatively by studying compliance with complete and correct tool entries.

Literature on the subject from a setting similar to ours is scarce. Narayanan $e t a l^{13}$ reported SATS tool use by nurses in a hospital in India, studied their undertriage and overtriage rates and found them to be in safe limits. Dalwai $e t$ $a l^{14}$ studied nurse triage ratings to analyse reliability and accuracy of tool in Pakistan and found it to be reliable. In another study in Afghanistan, Dalwai $e t$ al ${ }^{15}$ reported that tool application in mixed setting (ie, trauma and 


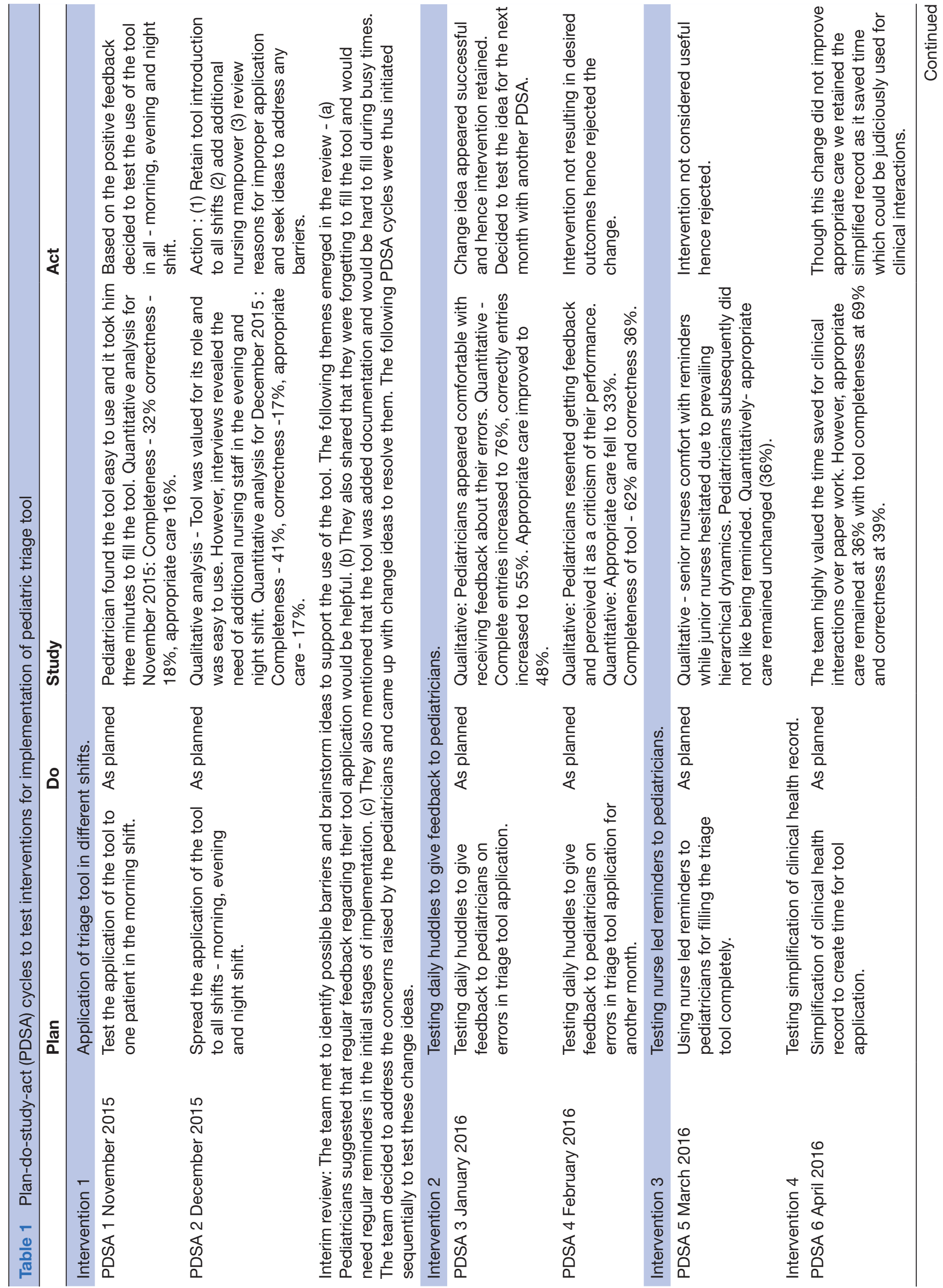




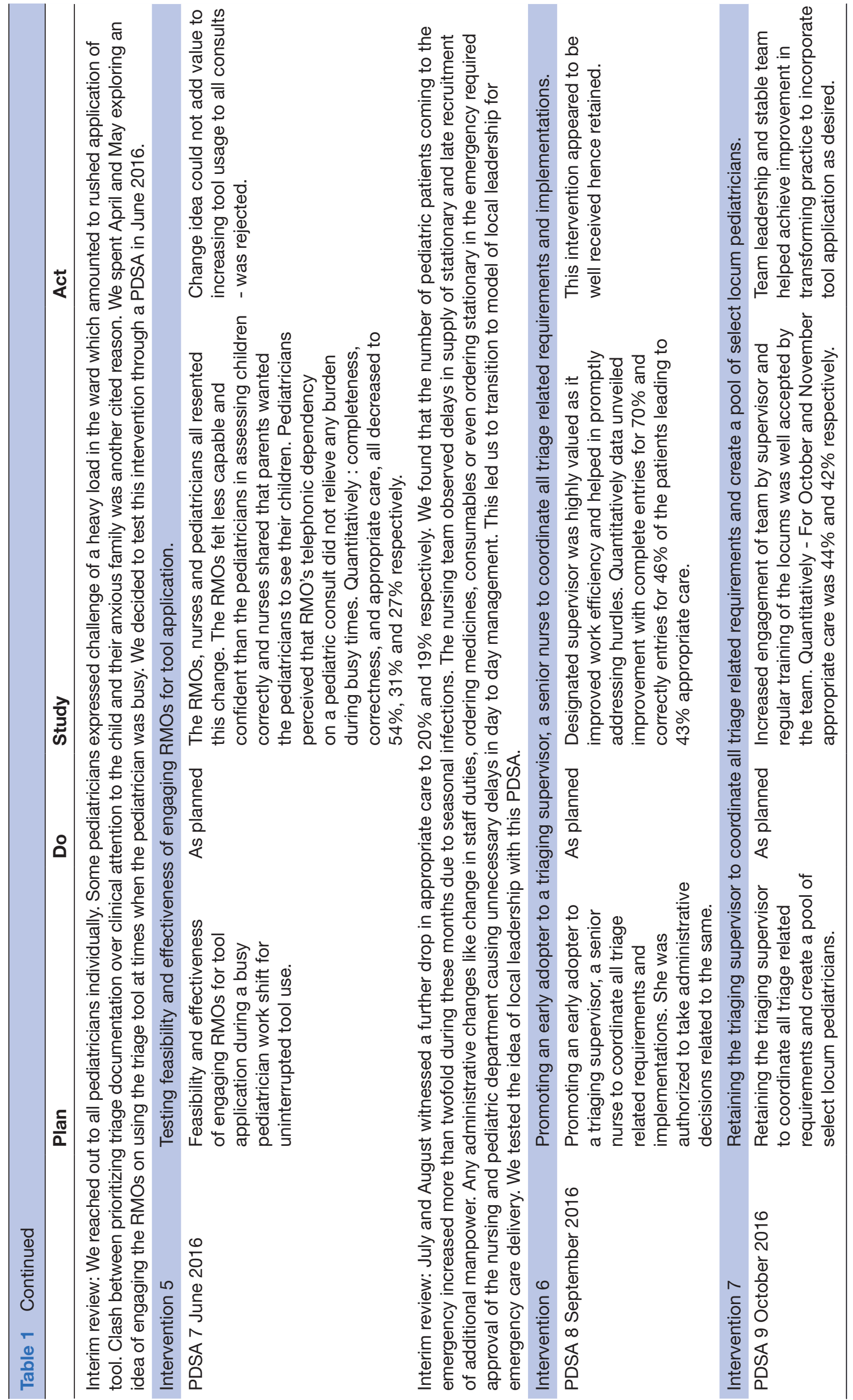


non-trauma illnesses) warrants context-specific assessments and incorporation of local conditions.

The study was successful in establishing a triage system for paediatric patients coming to the ED in a low and middle-income country. This is expected to encourage other care providers to expedite triaging into their health settings. We noted several unanticipated outcomes leading to rejection of several change ideas during our journey. The overall pursuit was still retained, keeping the larger objective in mind. This was attributed to the unique nature of the environment, the involvement of a team new to the improvement methodology and gaps in assessment of confounding variables.

\section{Lessons and limitations}

Many lessons were learnt during our journey. The first lesson was that just telling people to try something new even if they appreciate it (ie, use a new tool) does not work. It is pertinent to study the local work environment and team dynamics before introducing a change. In our case, the tool was introduced within the ED, comprised essentially of the RMOs and nurses who were assigned only to the ED. The paediatrician, on the other hand, worked at various other locations in the hospital and visited the ED intermittently. This compromised a sense of team belonging and ownership which fosters enhanced participation towards change in the environment. It was therefore important to understand and address such systemic challenges for successful implementation. Identifying a unifying force, a triaging supervisor in our case, helped align the nurses and paediatricians towards a common goal. Second, local ownership with autonomy to quickly make the required management decisions is critical. In our case, our existing management system in which emergency nurses had to seek approval for every minor change from nursing and paediatric department heads caused delays, confusion and fostered inefficiency. Giving autonomy to a senior nurse, a frontliner familiar with grass-roots challenges to take day-to-day decisions, was a major breakthrough. Third, the timing of introducing a change is critical. It is important to plan changes when work environment is receptive and participants can test and implement the change under regular circumstances. Trying new change ideas during the busy footfall phase received pushback from a lot of our staff. Another crucial learning was to have a contingency plan for special situations like high patient load during seasonal infections.

One of the main limitations is that we do not have documented data on the percentage of children requiring admission coming back to the outpatient department after being seen in the ED. However, on feedback from paediatricians we have found that they rarely encounter such patients. Another limitation was our inability to study a balancing measure. We anticipated that addition of the tool to the existing emergency care could increase time spent on one patient and contribute to delay in seeing subsequent patients and also delay in other tasks undertaken serially such as record disposal, patient counselling, transfer coordination, and so on. Given the multiplicity of tasks undertaken by nurses and paediatricians and the unpredictable nature of the work itself, creating a system of tracking time efficiently warranted use of technology such as bar coding which we did not possess. We were thus unable to objectively measure the imbalance generated from tool use towards other aspects of prevailing ecosystem which could potentially compromise our emergency care. However, qualitative data emerging from group discussions or interviews did not suggest such a concern.

Third, we could not explore patient feedbacks to assess patient-centric improvements.

Lastly, sustaining gains has been a significant challenge especially due to routine manpower attritions and busy workload seasons when a committed pool of locum paediatricians become impossible. As new recruits learn to adapt to needs of the new environment, tool being one of many, compliance with its correct application plunges. While local leadership proves instrumental at these times as a dedicated and reliable guiding presence, regular trainings and interactive feedbacks are also required to engage the team, which poses demands on the system. We continue to seek fresh inputs and feedbacks from new members to seek ways to address these limitations.

\section{CONCLUSION}

Triage is critical to emergency care but is underused in our facility as it is all over India. In this project we improved appropriateness of care by implementing a triage tool. Our first approach to introduce the tool and assume that it would work failed. Our second approach of identifying problems and using iterative tests to address these problems worked. We have sustained the improvement in appropriate care for 20 months. We suggest that other hospitals use our second approach and replicate triaging or other similar instruments of safe care. While choice of the instrument or change idea may be different, the method of embedding the change ideas remains the same.

Twitter Saru Bhartia @Saru4q

Acknowledgements We acknowledge the support and encouragement of $\mathrm{Mr}$ Abhishek Bhartia, director, and Dr Sneh Bhargava, medical director.

Contributors SB, NJ, JN and RW planned the original QI project, adapted and implemented the project and collected data. NJ drafted the paper with SB. All authors contributed to the manuscript and approved the final draft.

Funding The authors have not declared a specific grant for this research from any funding agency in the public, commercial or not-for-profit sectors.

Competing interests None declared.

Patient and public involvement Patients and/or the public were not involved in the design, or conduct, or reporting, or dissemination plans of this research.

Patient consent for publication Not required.

Ethics approval This project was deemed to be an improvement project and not a study on human subjects. Therefore, ethical improvement was not required.

Provenance and peer review Not commissioned; externally peer reviewed.

Data availability statement All data relevant to the study are included in the article or uploaded as supplementary information. 
Open access This is an open access article distributed in accordance with the Creative Commons Attribution Non Commercial (CC BY-NC 4.0) license, which permits others to distribute, remix, adapt, build upon this work non-commercially, and license their derivative works on different terms, provided the original work is properly cited, appropriate credit is given, any changes made indicated, and the use is non-commercial. See: http://creativecommons.org/licenses/by-nc/4.0/.

\section{REFERENCES}

1 Duke T, Cheema B. Paediatric emergency and acute care in resource poor settings. J Paediatr Child Health 2016;52:221-6.

2 Nolan T, Angos P, Cunha AJLA, et al. Quality of hospital care for seriously ill children in less-developed countries. The Lancet 2001;357:106-10.

3 World Health Organization. Paediatric emergency triage, assessment and treatment : care of critically-ill children. Geneva, Switzerland: World Health Organization, 2016: 88. https://www.who.int/maternal_ child_adolescent/documents/paediatric-emergency-triage-update/ en/

4 Mackway-Jones K, Marsden J, Windle J. Emergency triage: Manchester triage group. 3rd edn. Chichester: John Wiley \& Sons Ltd, 2014: 204.

5 Gilboy N, Tanabe T, Travers D. Emergency severity index (ESI): a triage tool for emergency department care. Rockville: Agency for healthcare research and quality, 2011. http://www. ahrq. gov/ research/esi/esihandbk.Pdf

6 Warren DW, Jarvis A, LeBlanc L, et al. Revisions to the Canadian triage and acuity scale paediatric guidelines (PaedCTAS). CJEM 2008;10:224-32.
7 Gerdtz MF, Collins M, Chu M, et al. Optimizing triage consistency in Australian emergency departments: the emergency triage education kit. Emerg Med Australas 2008;20:250-9.

8 World Health Organization. Emergency Triage Assessment and Treatment (ETAT):Manual for participants. Geneva, Switzerland: World Health Organization, 2005. https://apps.who.int/iris/bitstream/handle/ 10665/43386/9241546875_eng.pdf?sequence=1

9 Bhimani SA, Brown N, Mian Al. Streamlining pediatric emergency medicine at a tertiary-care hospital of a low- to middle-income country. Indian Pediatr 2015;52:1021-4.

10 Singhi S, Singhi S, Gupta G. Comparison of pediatric emergency patients in a tertiary care hospital vs a community hospital. Indian Pediatr 2004;41:67-72.

11 Mahajan P, Batra P, Shah BR, et al. The 2015 academic College of emergency experts in Indias Indo-US joint Working group white paper on establishing an academic department and training pediatric emergency medicine specialists in India. Indian Pediatr 2015;52:1061-71.

12 Jenson A, Hansoti B, Rothman R, et al. Reliability and validity of emergency department triage tools in low- and middle-income countries. Eur J Emerg Med 2018;25:154-60.

13 Narayanan UP, Padankatti S, Thomas K. Implementation of triage in a pediatric emergency department. Int J Emerg Med 2014;7:O3.

14 Dalwai MK, Twomey M, Maikere J, et al. Reliability and accuracy of the South African triage scale when used by nurses in the emergency department of Timergara Hospital, Pakistan. S Afr Med J 2014;104:372-5

15 Dalwai M, Valles P, Twomey M, et al. Is the South African triage scale valid for use in Afghanistan, Haiti and Sierra Leone? BMJ Glob Health 2017;2:e000160.

16 Anhøj J, Olesen AV. Run charts revisited: a simulation study of run chart rules for detection of non-random variation in health care processes. PLoS One 2014;9:e113825. 